\title{
Multimodal Imaging of Environmental and Biological Liquid Surfaces and Interfaces Using Imaging Mass Spectrometry
}

\author{
Xiao-Ying $\mathrm{Yu}^{1}$ \\ 1. Fundamental \& Computational Sciences Directorate, Pacific Northwest National Laboratory, \\ Richland, WA 99354, USA.
}

Imaging mass spectrometry (IMS), including time-of-flight secondary ion mass spectrometry (ToFSIMS), matrix-assisted laser desorption/ionization, or desorption electrospray ionization, uses the analytical power of mass spectrometry to generate chemical images illustrating the distribution of molecules in a sample. IMS generally requires that the analyte be transferred from the condensed phase, ionized, separated in vacuum, and detected. Imaging liquid surfaces and interfaces is a known challenge due to the high volatility of liquids in vacuum.

We developed a vacuum compatible microfluidic interface, System for Analysis at the Liquid Vacuum Interface (SALVI), to enable surface analysis of liquids and liquid-solid interactions using ToF-SIMS. SALVI's detection window is an aperture of 2-3 $\mu \mathrm{m}$ in diameter open to vacuum, which allows direct detection of the liquid surface. Liquid is withheld by surface tension within the aperture. SALVI is composed of a silicon nitride (SiN) membrane and polydimethylsiloxane (PDMS) microchannel in a block [1, 2]. Its applications to enable liquid ToF-SIMS as a new analytical tool were evaluated and reported. Most recently, we showed that solvent structure can be determined. Moreover, we demonstrated in situ correlative imaging of single mammalian cells using ToF-SIMS guided by structure illumination microscopy (SIM) for the first time. In addition, we extended SALVI for biofilm growth and real-time characterization [3]. We provided the first correlative chemical imaging of live biofilms using confocal laser scanning microscopy, nuclear magnetic resonance imaging, and ToF-SIMS.

A ToF-SIMS V spectrometer (IONTOF GmbH, Münster, Germany) was used mainly in these experiments. A pulsed $25 \mathrm{keV} \mathrm{Bi}_{n}{ }^{+}$(beam size: $\sim 250 \mathrm{~nm}$ ) ion beam with an incident angle of 45 degree off the normal was used as the primary ion beam for all measurements. The SIMS measurements were performed at the beam current of $\sim 1.0$ pA with a beam width of 130 ns and a repeated frequency of 20 $\mathrm{kHz}$. The main chamber vacuum pressure was $2-4 \times 10^{-7}$ mbar with SALVI inside the chamber, and the pressure only slightly increased to $3-5 \times 10^{-7}$ mbar during measurements. This indicates that no spraying or fast spreading of aqueous solutions occurs through the aperture.

Figure 1 depicts the results of the ionic liquid solvent structure observation using SALVI and in situ liquid ToF-SIMS. For ionic liquids with different $\mathrm{CO}_{2}$ loadings, the $\mathrm{m} / \mathrm{z}$ spectra show clear spatial chemical inhomogeneity as predicted by molecular dynamic simulations. Figure 2 depicts the first correlative imaging results of a mouse lung C10 cell using SIM and ToF-SIMS. These new results demonstrated advancements of multimodal mesoscale imaging of liquid surfaces and liquid-solid interfaces in situ in their natural liquid environment using a universal sample holder, SALVI [4].

References:

[1] L Yang et al., J. Vac. Sci. Technol. A 29 (2011), art. no., 061101, doi: 10.1116/1.3654147.

[2] L Yang et al., Lab Chip 11 (2011), 2481-4. doi: 10.1039/c01c00676a. 
[3] H Xin et al., Analyst (2014), 139(7), 1609-13. doi: 10.1039/C3AN02262E.

[4] H Shi et al., Catal. Sci. Technol. (2015), advanced on-line. doi: 10.1039/C4CY01720J.

[5] The authors acknowledge funding from the Pacific Northwest National Laboratory (PNNL) Technology Development Program, Materials Synthesis and Simulation across Scales $\left(\mathrm{MS}^{3}\right)$ Initiative and the Chemical Imaging Initiative (CII) Laboratory Directed Research and Development fund.
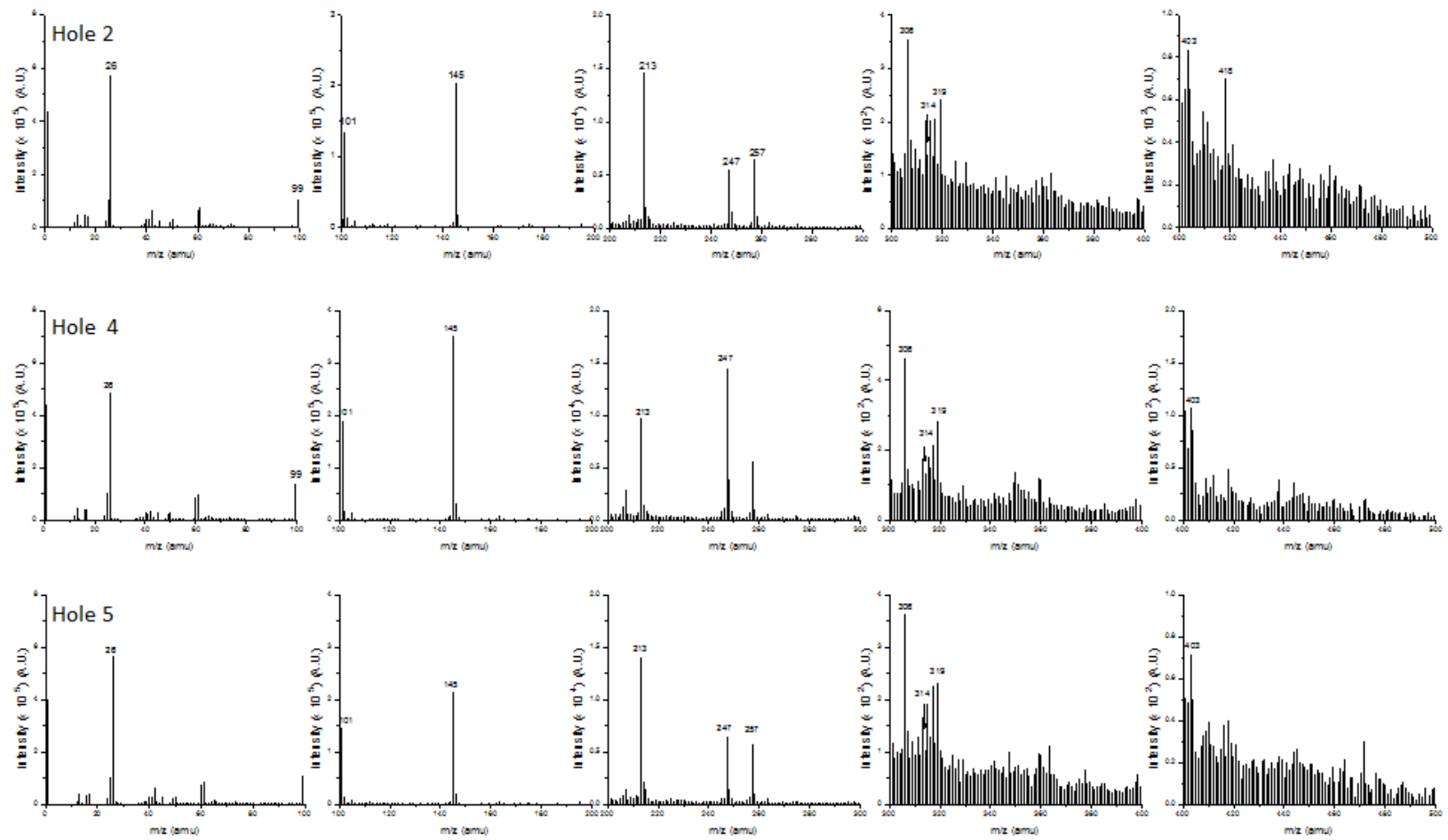

Figure 1. Negative $\mathrm{m} / \mathrm{z}$ spectra of an ionic liquid of $100 \% \mathrm{CO}_{2}$ loading at different locations along the microchannel.

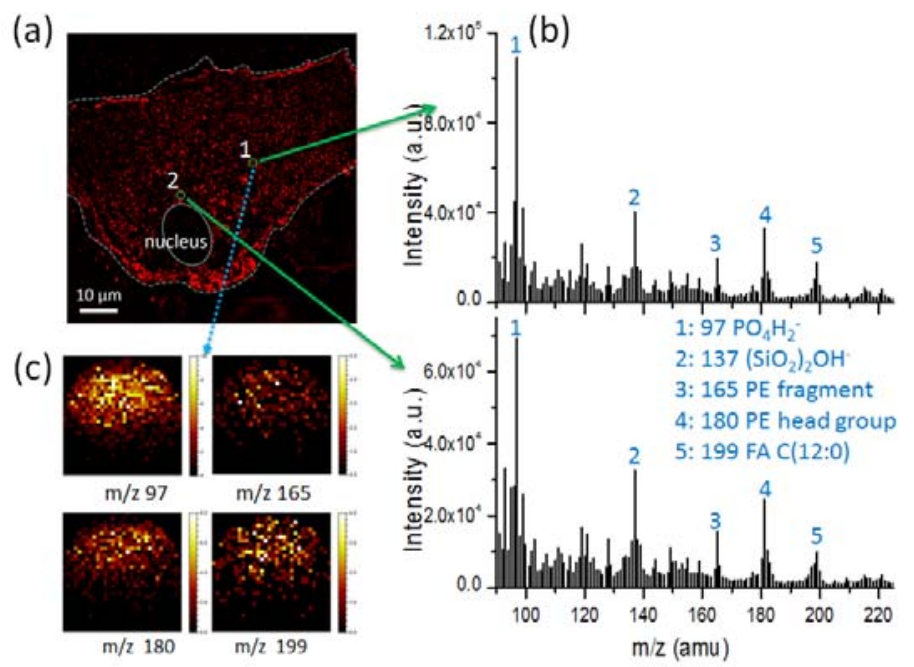

Figure 2. (a) Fluorescence SIM image of a C10 cell in SALVI. (b) ToF-SIMS m/z spectra acquired at the corresponding locations highlighted in green circles in (a); and (c) 2D ToF-SIMS images showing the spatial distribution of representative cell molecular fragments from location 1 in (a). 